\title{
Novel Solutions to Low-Frequency Problems With Geometrically Designed Beam-Waveguide Systems
}

\author{
W. A. Imbriale, M. S. Esquivel, and F. Manshadi \\ Communications Ground Systems Section
}

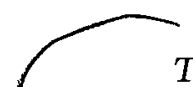

The poor low-frequency performance of geometrically designed beam-waveguide ( $B W G$ ) antennas is shown to be caused by the diffraction phase centers being far from the geometrical optics mirror focus, resulting in substantial spillover and defocusing loss. Two novel solutions are proposed: (1) reposition the mirrors to focus low frequencies and redesign the high frequencies to utilize the new mirror positions and (2) redesign the input feed system to provide an optimum solution for the low frequency. A novel use of the conjugate phase-matching technique is utilized to design the optimum low-frequency feed system, and the new feed system has been implemented in the JPL research and development BWG as part of a dual $S$ - $/ X$-band $(2.3-G H z / 8.45-G H z)$ feed system. The new $S$-band feed system is shown to perform significantly better than the original geometrically designed system.
\end{abstract}

\section{Introduction}

JPL has recently built a new 34-m beam-waveguide (BWG) antenna at Goldstone's Deep Space Station 13 site (DSS 13). The design of the center-fed BWG consists of a beam magnifier ellipse, in a pedestal room located below ground level, that transforms a 22-dB gain feedhorn into a high-gain 29-dB gain pattern for input to a standard four-mirror (two flat and two paraboloid) BWG system. The design of the upper section of the BWG is based on geometrical optics (G.O.) criteria introduced by Mizusawa and Kitsuregawa in 1973 [1,2], which guarantees a perfect image from a reflector pair. The system was initially designed (Phase 1) for operation at $8.45 \mathrm{GHz}$ (X-band) and $32 \mathrm{GHz}$ (Ka-band) and has less than 0.2-dB loss (determined by comparing the gain of a 29-dB gain horn feeding the dual-shaped reflector system with that obtained using the BWG system) [3,4]. In Phase 2, S-band (2.3 GHz) is to be added.

If a standard 22-dB S-band horn is placed at the input focus of the ellipse, the BWG loss is greater than $1.5 \mathrm{~dB}$, primarily due to the fact that, for low frequencies, the diffraction phase centers are far from the G.O. mirror focus, resulting in a substantial spillover and defocusing loss. This defocusing is especially a problem for the magnifier ellipse, where the S-band phase center at the output of the ellipse is $3 \mathrm{~m}$ from the G.O. focus. If the input to the paraboloids were focused, the output defocusing would only cause a $0.3-\mathrm{dB}$ loss. One solution would be to move the high-frequency phase center at the ellipse output to the low-frequency phase center (accomplished at X-band by simultaneously increasing the gain of the input horn to $26 \mathrm{~dB}$ and moving the horn phase center $0.5-\mathrm{m}$ below the input focus) and to reposition the phase centers to the input focus of the paraboloids. This can be accomplished by leaving the ellipse 
in its original position and increasing the spacing between the paraboloids. With this arrangement, the BWG loss at S-band is only $0.4 \mathrm{~dB}$ and the loss at $\mathrm{X}$-band is virtually unaffected. This solution has the disadvantage, however, of necessitating a physical modification to the structure of the BWG system.

A second solution is to redesign the horn to provide an optimum solution for S-band. The question is how to determine the appropriate gain and location for this feed. A straightforward design by analysis would prove cumbersome because of the large number of scattering surfaces required for the computation. Rather, a unique application was made of the conjugate phase-matching techniques to obtain the desired solution. A plane wave was used to illuminate the main reflector, and the fields from the currents induced on the subreflector were propagated through the BWG to a plane centered on the input focal point. By taking the complex conjugate of the currents induced on the plane and applying the radiation integral, the far-field pattern was obtained for a theoretical horn that maximizes the antenna gain.

To synthesize a horn quickly and inexpensively, the theoretical horn was matched as well as possible by an appropriately sized circular corrugated horn. The corrugated horn performance was only 0.2 -dB lower than the optimum theoretical horn but 1.4-dB above the standard 22-dB horn. A system employing the corrugated horn was built and tested and installed in the 34-m BWG antenna as part of a simultaneous $\mathrm{S}-/ \mathrm{X}$-band receiving system.

\section{The Problem}

The basic design of the center-fed beam waveguide is shown in Fig. 1. The shaped dual-reflector system (focal point $f_{1}$ ) is designed to provide uniform illumination with a 29.8-dB-gain horn at the input. The upper four mirrors of the beam waveguide (from $f_{2}$ to $f_{1}$ ) are designed to image the input (at $f_{2}$ ) to the output $\left(\right.$ at $\mathrm{f}_{1}$ ). Thus, to provide a 29.8 -dB-pattern output at $\mathrm{f}_{1}$ requires a 29.8 -dB-gain pattern at the input, $f_{2}$. The 29.8-dB-gain pattern is generated by using a 22-dB-gain horn at $f_{3}$ (the input focus of the magnifier ellipse) to provide the required gain at the output focus of the ellipse, $f_{2}$. Figure 2 compares the input and output patterns from the BWG system with the 29-dB-gain horn at X-band. Since the BWG project seeks to introduce S-band $(2.3 \mathrm{GHz})$ into the antenna in the Phase 2 project, it is useful to inquire what happens when a 22-dB S-band horn is placed at the input focus of the ellipse. Ignoring spillover past the BWG mirrors, the defocusing loss is $0.9 \mathrm{~dB}$. The BWG spillover loss is $0.5 \mathrm{~dB}$, yielding a total BWG loss of $1.4 \mathrm{~dB}$. The principal cause of the defocusing loss is related to the fact that, for low frequencies, the diffraction phase center at the Cassegrain focus $\mathrm{f}_{1}$ is far-3.56 $\mathrm{m}$ (140 in.) - from the G.O. focus. This loss is illustrated in Fig. 3, where a plot of gain versus the z-displacement motion of the BWG assumes that the entire BWG is moved relative to the focal point of the dual-reflector system at $\mathrm{f}_{1}$. Only the aperture illumination, phase efficiency, dual-reflector spillover, and center blockage loss are included in the calculation; BWG internal spillover is ignored for this comparison since it would be the same for each point of the curve in Fig. 3. This defocusing is especially a problem for the magnifier ellipse, where the S-band phase center at the output of the ellipse is $3.05 \mathrm{~m}\left(120 \mathrm{in}\right.$.) from the G.O. focus at $\mathrm{f}_{2}$. Thus, the input to the two-paraboloid section is defocused, causing the majority of the spillover loss and adding to the defocusing of the paraboloid output. If the input to the upper BWG section were focused, the output would then be defocused by some 1.5 to $2.25 \mathrm{~m}$. However, this defocusing would cause only a 0.2- to 0.3-dB loss. Efforts were made to determine if adjustment to the input pattern amplitude or phase would move the low-frequency diffraction phase center to the G.O. phase center at $f_{2}$ [5]. It was determined that if the ellipsoidal mirror were large enough $(>30 \lambda)$ it would be possible, but for smaller ellipsoids ( $18 \lambda$ in this case), it was not possible to move the focus all the way to the G.O. phase center, $f_{2}$.

\section{Optics Redesign}

To overcome the problem of the disparate phase centers (between X-and S-bands), it was found that, instead of moving the low-frequency phase center to the G.O. focus for the ellipse, the high-frequency 


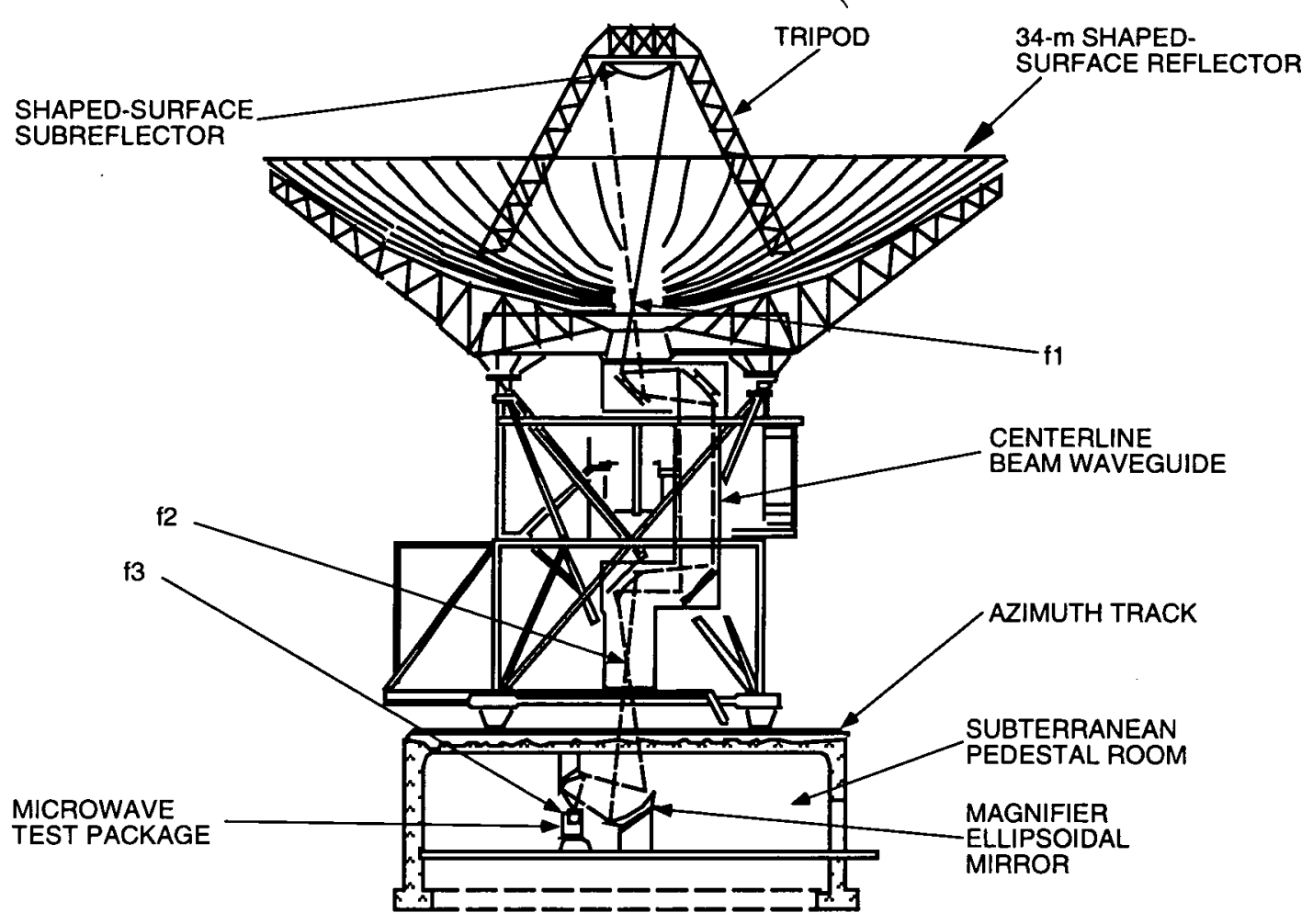

Fig. 1. New NASA beam-waveguide antenna.

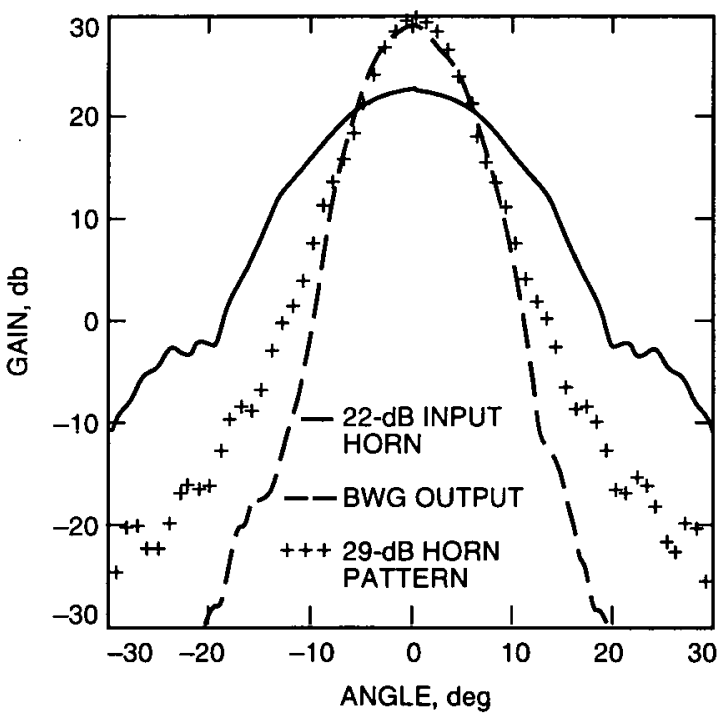

Fig. 2. Beam-waveguide input and output radiation patterns at $X$-band.

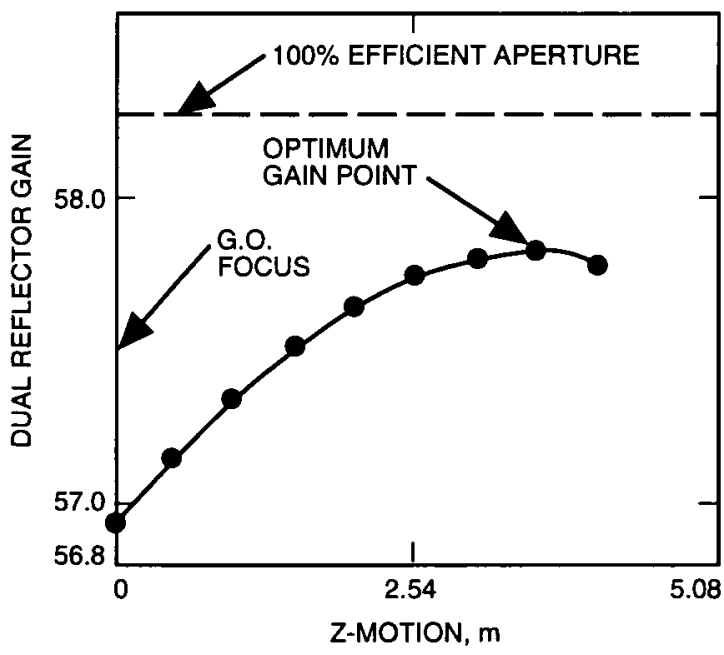

Fig. 3. Beam-waveguide defocusing curve at S-band.

phase center should be moved to the low-frequency phase center and the ellipse output repositioned to put these phase centers at the input focus of the paraboloids. The motion of the X-band phase center can be accomplished by simultaneously increasing the gain of the X-band input horn to $26 \mathrm{~dB}$ and moving the horn phase center approximately $0.5-\mathrm{m}$ below the input focus at $\mathrm{f}_{3}$. Since the position of the pedestal room is fixed with respect to the reflector, and since there is insufficient room to move the output focal point of the ellipse the required distance upward, the separation of the parabolas is increased to effectively move the paraboloid input focus down to the phase centers (see Fig. 4). Because the motion of the paraboloids 
is in the path where the rays are parallel, and the increase in distance is not sufficient for the rays to diverge, there is very little effect on the BWG performance. The distance selected was $2.03 \mathrm{~m} \mathrm{(80} \mathrm{in.),}$ to minimize the motion of the X-band phase center, so consequently a small S-band defocusing loss is retained. With this arrangement, the BWG loss at S-band is only $0.4 \mathrm{~dB}$, and the BWG loss at X-band is virtually unaffected. Figure 5 compares the S- and X-band BWG output with the 29-dB horn and indicates that the modified design is an acceptable compromise.

The analysis of the RF performance is calculated using physical optics (P.O.) on the BWG mirrors and subreflector [6] and the Jacobi-Bessel series [7] on the main reflector. In these calculations, a feed radiation pattern was modeled as a set of spherical-wave expansion (SWE) coefficients expanded about $f_{3}[8]$. The coefficients were used to illuminate $M_{5}$, the BWG mirror in the pedestal room. The induced currents on $M_{5}$ were cascaded by means of P.O. through $M_{4}, M_{3}, M_{2}, M_{1}$, the subreflectors, and the main reflectors. The Jacobi-Bessel method was implemented at the main reflector to obtain the secondary pattern of the antenna.

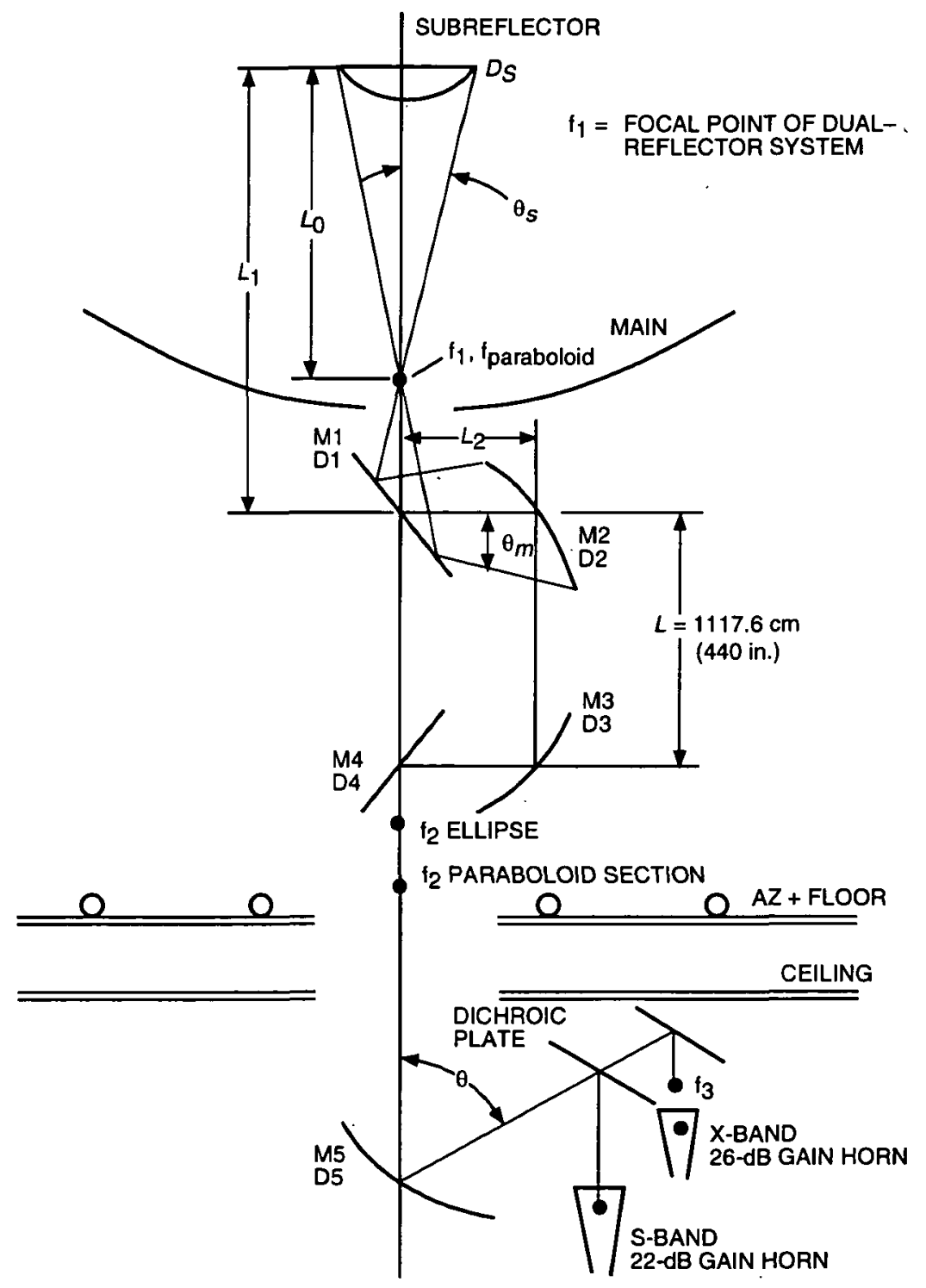

Fig. 4. Modified design geometry. 


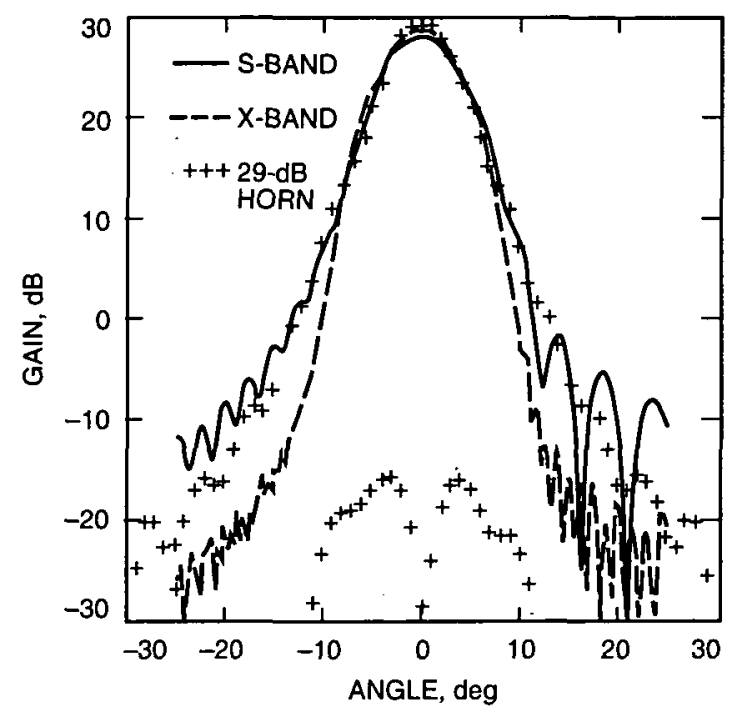

Fig. 5. Modified design performance (output of BWG system).

Even though the mirrors are unaffected, this solution still requires a physical modification to the BWG structure and was deemed unacceptable; thus, another method that did not modify the BWG system itself was required.

\section{Focal-Plane Method}

The goal of the design was to maximize the gain over noise temperature (G/T) of the BWG antenna. Since there are a large number of scattering surfaces (eight total), an optimization method that required repeated computation of the gain and noise temperature of the entire system would be rather time consuming. Instead, a unique application 'of the conjugate phase-matching technique (called the focalplane method) was tried. In this method, a uniform plane wave was used to illuminate the main reflector, and the fields from the currents induced on the subreflector were propagated through the BWG, $M_{1}, M_{2}$, $\mathrm{M}_{3}, \mathrm{M}_{4}$, and $\mathrm{M}_{5}$. Finally, the currents on a circular aperture with a $23 \lambda$ diameter at the focal plane centered at $f_{3}$ (Fig. 6) were computed. By taking the complex conjugate of these currents and applying the radiation integral, the far-field pattern was obtained for a theoretical horn that should maximize the gain. There was no a priori guarantee that the pattern produced by this method would be easily realized. However, the pattern is nearly circularly symmetric, and the theoretical horn was able to be matched fairly well by a circular corrugated horn.

Figure 7 shows the near-field E-plane patterns of the theoretical horn and a 19-dBi circular corrugated horn. The agreement in amplitude and phase is quite good out to $\theta=21 \mathrm{deg}$, the angle subtended by $\mathrm{M}_{5}$. The point of reference for the SWE coefficients used to generate the 19-dBi corrugated horn pattern was shifted until the radiation pattern matched the one of the theoretical horn centered at the focal plane $\mathrm{f}_{3}$. By this method, the position of the 19-dBi corrugated horn in the antenna could be determined. It turned out that the S-band corrugated horn's aperture position was $352.425 \mathrm{~cm}$ from the center of the magnifying ellipsoid $\mathrm{M}_{5}$.

The 19- $\mathrm{dBi}$ circular corrugated horn pattern was converted into a set of SWE coefficients that was then used in the P.O. analysis of the 34-m BWG antenna at S-band. Figure 8 shows the input and output of the magnifying ellipse, $M_{5}$, along with the output of the BWG system. The 19-dB pattern of 


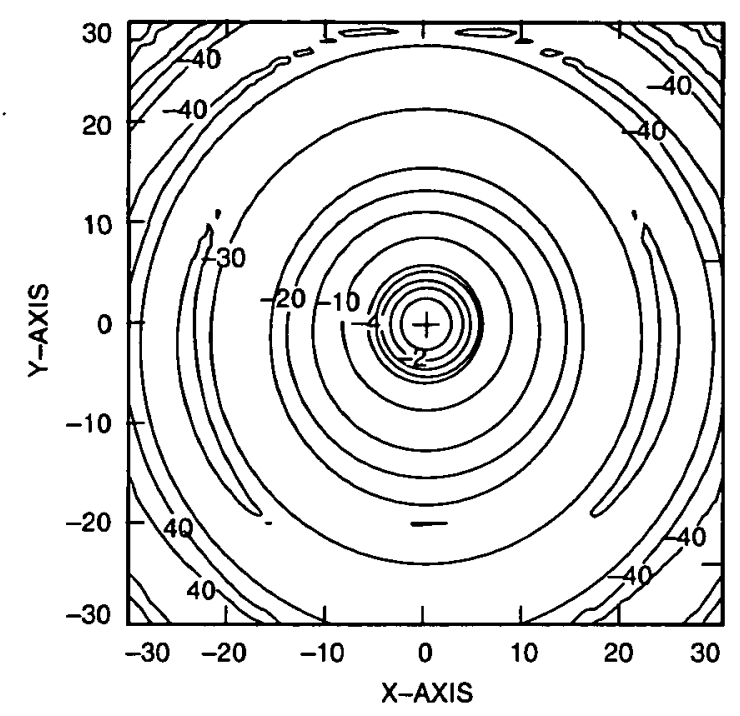

Fig. 6. Contour plot of currents induced on plane located at $f_{3}$ using the focal-plane method.

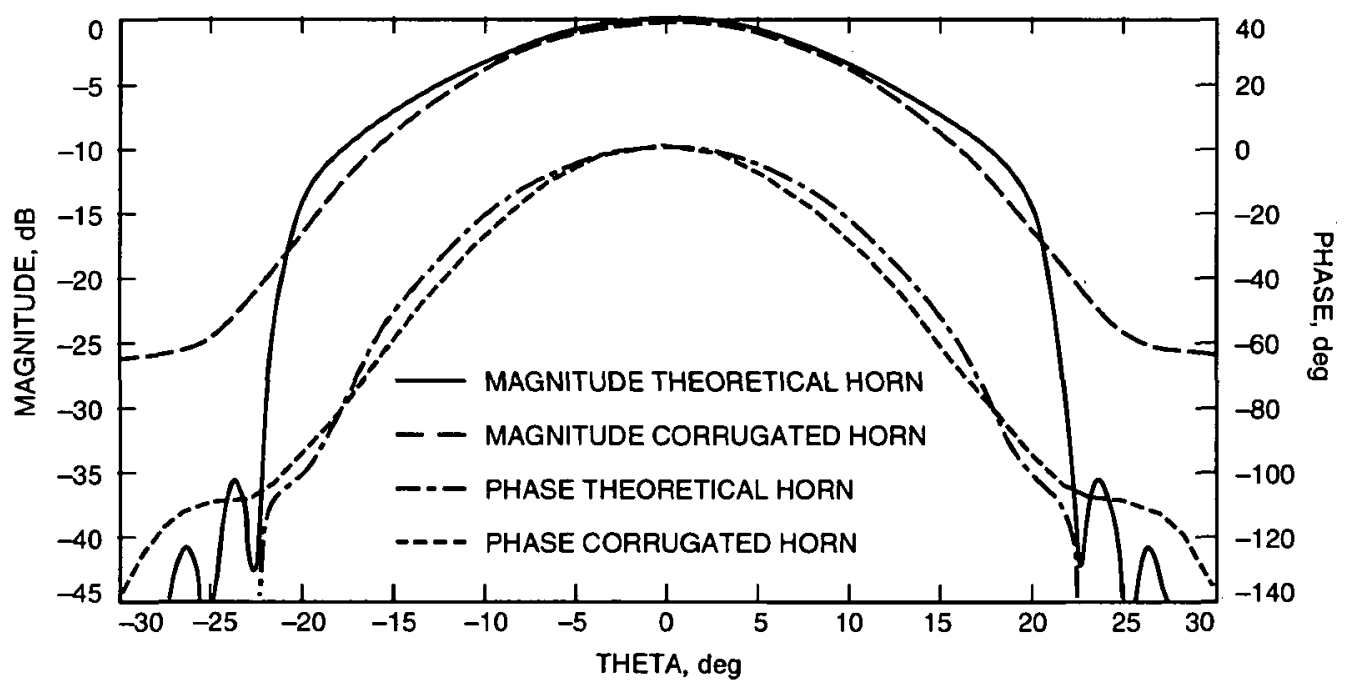

Fig. 7. E-plane near-field $\left(R=\mathbf{4} .2 \mathrm{~m}\right.$, referenced to $\left.f_{3}\right)$ patterns.

the corrugated horn is magnified into a $28.7-\mathrm{dB}$ pattern by the ellipse; the BWG mirrors add an extra $1.1 \mathrm{~dB}$, so that at the output of the system, the gain of the pattern is $29.8 \mathrm{~dB}$, the gain from which the dual-shaped system was synthesized.

Basically, the focal-plane method provided an unexpected solution to the defocusing problem of the 34-m BWG antenna at S-band: the use of a lower-gain horn. Previous work done on the antenna at $\mathrm{X}$-band and $\mathrm{Ka}$-band had shown that its $\mathrm{G} / \mathrm{T}$ would improve if corrugated horns with higher gains than the original-design $22 \mathrm{~dB}$ were used. For instance, an X-/Ka-band feed system uses corrugated horns with gains of 25.0 and $26 \mathrm{~dB}$, respectively [9]. Thus, when the task of implementing an S-band feed system in the antenna was initiated, a solution that required a higher-gain horn was expected.

Part of the skepticism was in the area of noise temperature. It was well known that a lower-gain horn would contribute more spillover, which would increase the noise temperature of the system. What was 
not understood at the time was that the 19-dBi corrugated horn would only have a higher spillover loss at the first reflector, $\mathrm{M}_{5}$, and that its performance through the remainder of the BWG system would be better than for the standard 22-dBi corrugated horn. Table 1, which lists P.O. and Jacobi-Bessel analysis results of the antenna at S-band, corroborates this observation. In this table, the spillover of the antenna mirrors, the antenna efficiency, and system noise temperature are listed for the 19-dBi corrugated horn and the theoretical horn pattern predicted by the focal-plane method. Also, for comparison purposes, the calculated performance of a 22-dBi corrugated horn is presented from [10].

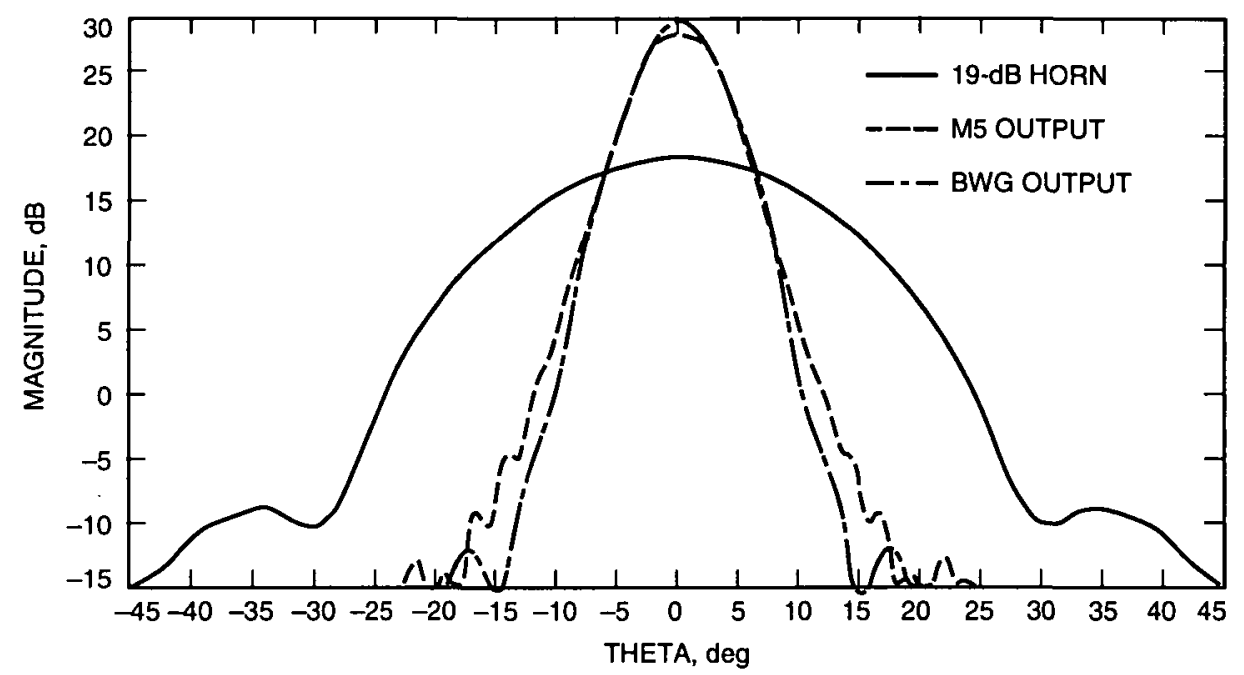

Fig. 8. Beam-waveguide input and output radiation patterns at S-band.

Table 1. S-band P.O. and Jacobi-Bessel calculations.

\begin{tabular}{|c|c|c|c|}
\hline Parameter & $\begin{array}{l}\text { 22-dBi corrugated } \\
\text { horn [10] }\end{array}$ & $\begin{array}{c}\text { 19-dBi corrugated } \\
\text { horn }\end{array}$ & $\begin{array}{c}\text { Theoretical } \\
\text { horn }\end{array}$ \\
\hline \multicolumn{4}{|l|}{ Spillover, percent } \\
\hline $\mathrm{M}_{6}$ & - & 0.41 & - \\
\hline $\mathrm{M}_{5}$ & 2.05 & 2.46 & 0.24 \\
\hline $\mathrm{M}_{4}$ & 1.57 & 0.70 & 1.19 \\
\hline $\mathrm{M}_{3}$ & 5.91 & 0.73 & 0.86 \\
\hline $\mathrm{M}_{2}$ & 5.55 & 0.96 & 1.29 \\
\hline $\mathrm{M}_{1}$ & 1.36 & 0.26 & 0.46 \\
\hline Subreflector & - & 1.14 & 1.94 \\
\hline Main reflector & - & 0.94 & 3.61 \\
\hline \multicolumn{4}{|l|}{ Efficiency } \\
\hline Total efficiency & 0.48415 & 0.6827 & 0.69502 \\
\hline Total efficiency, dB & 55.102 & 56.594 & 56.672 \\
\hline \multicolumn{4}{|l|}{ Noise temperature } \\
\hline Total noise, $\mathrm{K}$ & 73.574 & 37.10 & 35.314 \\
\hline Total noise, $\mathrm{dB}$ & 18.67 & 15.69 & 15.48 \\
\hline $\mathrm{G} / \mathrm{T}, \mathrm{dB}$ & 36.43 & 40.90 & 41.19 \\
\hline
\end{tabular}




\section{BWG S-/X-Band Feed System}

The S-band feed is part of a simultaneous S-/X-band receive system implemented on the new BWG antenna. The general configuration of the feed system, the detail design, and measured performance are described in this section.

\section{A. Theory of Operation}

Figure 9 shows the main components of the S-/X-band feed system: the X-band feed, the S-band feed, the S-/X-band dichroic reflector, and the X-band flat reflector. The S-band receive frequency band is 2200 to $2300 \mathrm{MHz}$, and the X-band receive frequency band is 8200 to $8600 \mathrm{MHz}$.

The S-band signal received from deep space is collected by the main/subreflector and is focused at $f_{1}$. Reflectors $M_{1}$ through $M_{4}$ guide the signal to the rotating ellipsoid focus $\mathrm{f}_{2}$. The signal is then scattered off the ellipsoid mirror, reflected by the dichroic reflector, and focused at the other focal point of the elliptical mirror. This signal is received by the S-band feedhorn in the S-band feed package.

The X-band signal is guided by the beam waveguide to the basement in the same manner as the S-band signal. However, after scattering off the ellipsoid, it passes through the dichroic mirror with very little loss, reflected by the X-band flat reflector, and is focused at the other focal point of the ellipsoid. This signal is received by the $\mathrm{X}$-band feedhorn in the $\mathrm{X}$-band package.

\section{B. Detail Design}

A block diagram of the S-/X-band feed packages is provided in Fig. 10. The low-noise amplifier (LNA) is a dual-frequency LNA, i.e., it contains both an X-band LNA and an S-band LNA in one cryogenic package. The S- and X-band feeds are packaged separately; however, they are physically connected since they share the same LNA package. The feedhorns are corrugated with the same corrugations and flare angle as the standard JPL feedhorns [11]. The gain of the feedhorns is $19.1 \mathrm{~dB}$ for S-band and $25.0 \mathrm{~dB}$ for X-band. As discussed in the previous section, a 25-dB horn was used at X-band instead of the originally designed system (using the $22-\mathrm{dB}$ horn) since it was discovered that a $25-\mathrm{dB}$ horn would reduce the noise temperature. Although the $25-\mathrm{dB}$ horn reduced the efficiency somewhat, the gain in noise temperature more than offset the loss in efficiency, and the 25-dB-gain horn maximized the G/T of the system. Right-hand circular polarization (RCP) or left-hand circular polarization (LCP) polarizers provide the capability to select the reception. In the S-band package, the position of the polarizer can be changed easily because of the use of the rotary joints, but in the X-band package, the position of the polarizer is fixed. To change polarization on the X-band, the polarizer has to be unscrewed and then rotated. The couplers are used for injection of noise to check the linearity of the LNAs. The waveguide switches are used to connect the LNAs to the feedhorns or to the ambient loads for noise temperature and linearity measurements.

The S-/X-band dichroic reflector is a frequency selective surface that passes the X-band signal but reflects the S-band signal. The S-/X-band dichroic plate used at DSS 13 is a $198.1-\mathrm{cm} \times 141.5-\mathrm{cm}$ $\times 3.576-\mathrm{cm}$ rectangular aluminum plate with an elliptical perforated area (see Fig. 11). The holes in the perforated area are based on an old dichroic plate design [12]. This design employs the Pyleguide holes originally used by Pyle [13]. However, to reduce the fabrication cost, the corner radius of the holes was increased from 0.013 to $0.318 \mathrm{~cm}$, as shown in Fig. 11. An analysis of the propagation constant of the fields in the Pyleguide holes shows that the change in the propagation constant due to this modification is far less than the change due to the tolerances of the other critical dimensions of the holes. ${ }^{1}$ This minor change reduced the fabrication cost of the S-/X dichroic reflector by more than 60 percent.

\footnotetext{
1 J. C. Chen and P. H. Stanton, "Effect of Corner Radius on the Performance of an S-/X-Band Dichroic Plate With Pyleguide Aperture," JPL Interoffice Memorandum 3327-92-078 (internal document), Jet Propulsion Laboratory, Pasadena, California, November 24, 1992.
} 


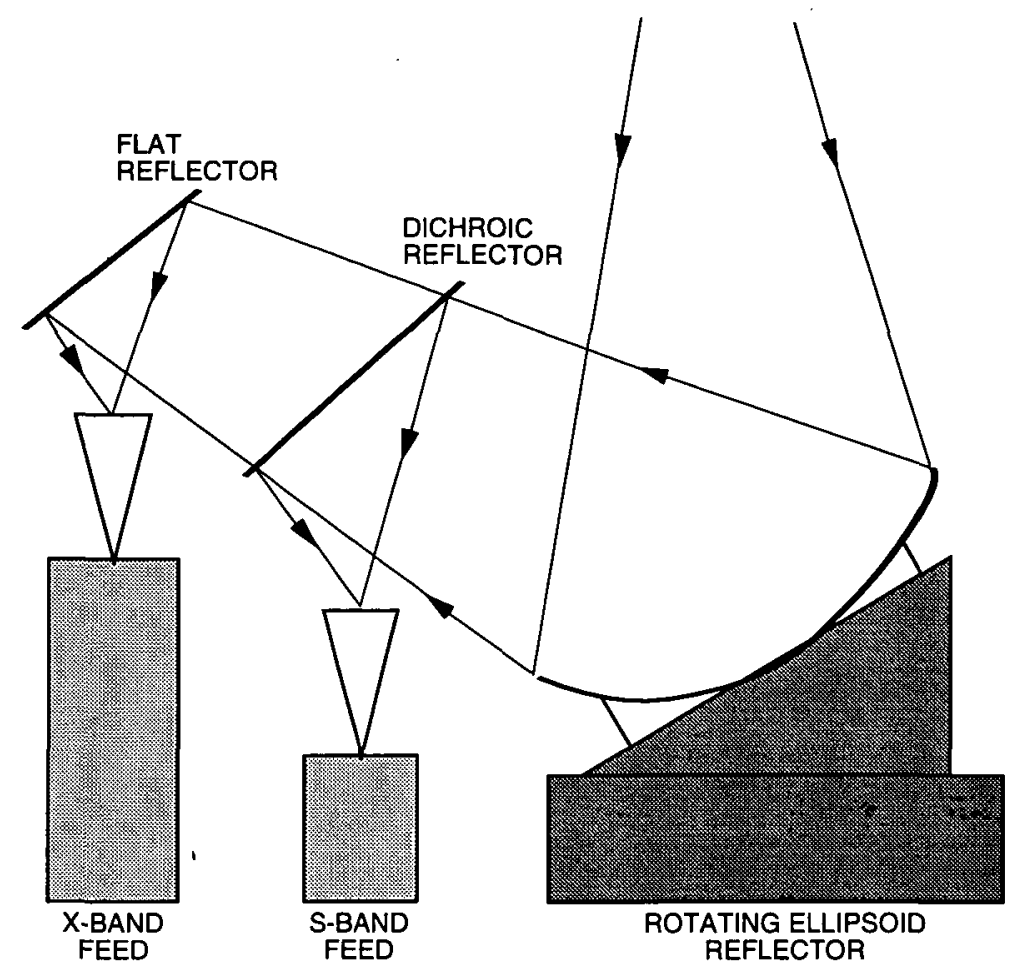

Fig. 9. DSS-13 S-/X-band feed system.
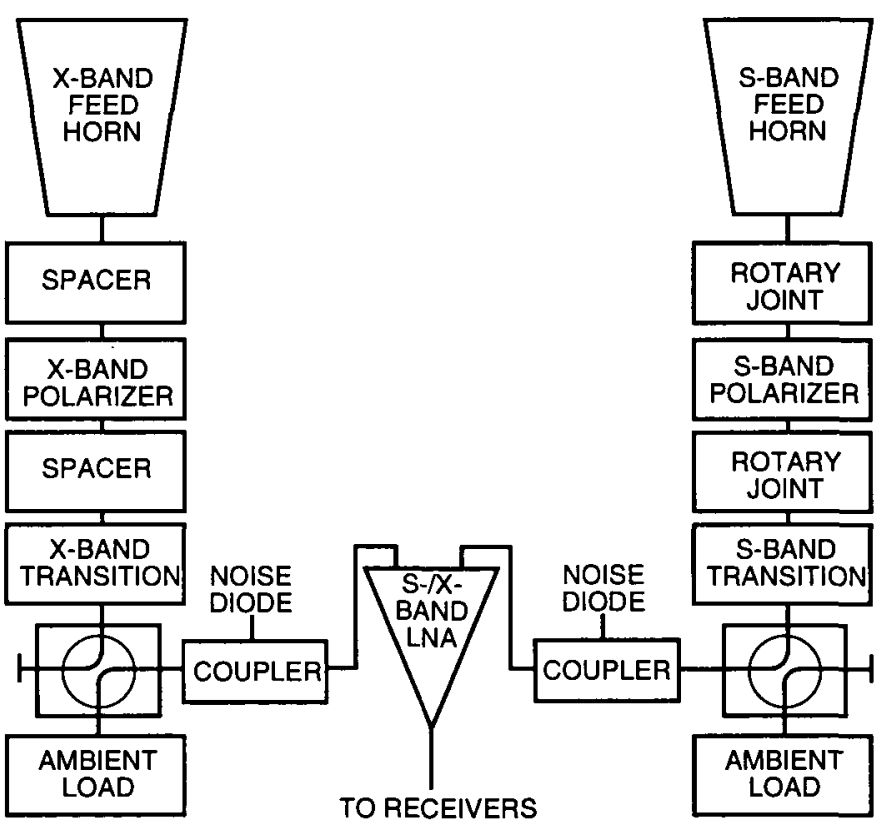

Fig. 10. DSS-13 S-/X-band feed block diagram.

The frames for the S- and X-band packages were fabricated using Bosch extruded aluminum struts. These struts are prefabricated, strong, lightweight, and flexible. Their anodized aluminum surface finish is scratch and corrosion resistant. Since all the elements of the frames are bolted together, it is very easy to modify these frames as needed in the future. The use of these materials resulted in a cost savings of more than 50 percent compared to conventional welded steel framing. 

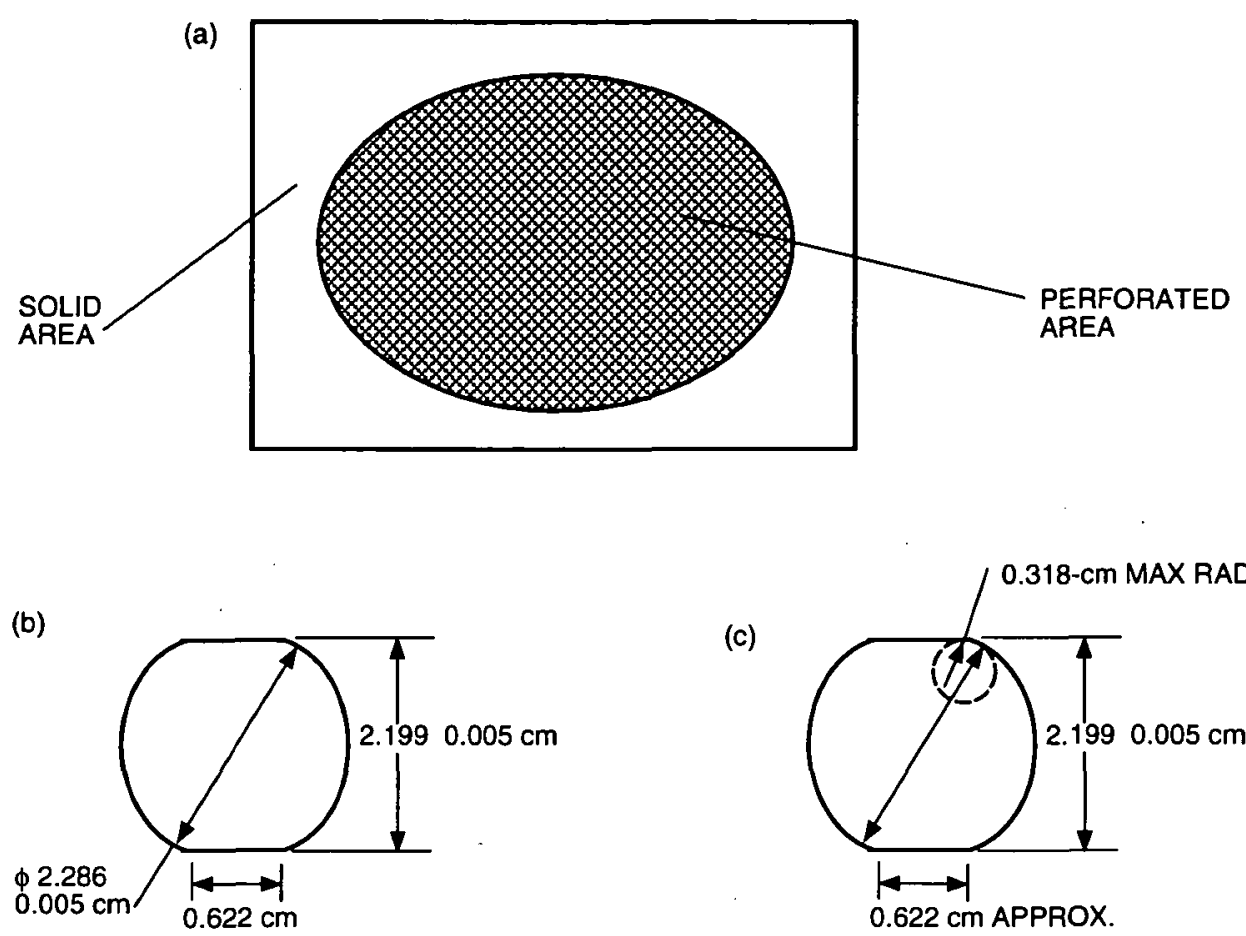

(c)

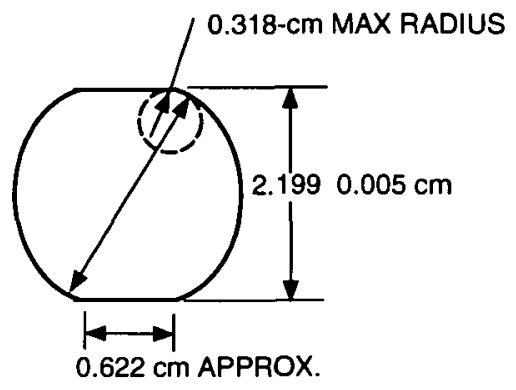

Fig. 11. DSS-13 S-/X-band dichroic reflector: (a) layout, (b) original Pyleguide design, and (c) modified Pyleguide design.

Figure 12 shows a picture of the S- and X-band microwave feed assembly installed in the DSS-13 BWG antenna.

\section{Feed System Performance}

The predicted and measured noise temperatures of the S-/X-band LNAs, microwave feeds, and the overall DSS-13 BWG antenna are shown in Table 2. The higher-than-standard DSN noise temperature measured for the X-band LNA is due to the age of the package, which was acceptable for its intended use. The predicts are calculated from the theoretical or measured loss of the individual component of each system. The measurements for the feeds were made at Goldstone before installation in the antenna pedestal room. The measurements for the overall antenna were made after the feed packages were installed and aligned in the pedestal room.

The predicted S-band efficiency from Table 1 was 68 percent, and the measured efficiency was 67.5 percent, demonstrating the successful design and implementation. For comparison, the predicted X-band efficiency (at the rigging angle of $45 \mathrm{deg}$ ) was 72.7 percent, and the measured efficiency, including the dichroic plate, was 70.1 percent.

There are two interesting observations on the variation of efficiency with azimuth and elevation. The main reflector surface shape changes slightly with elevation due to backup structure distortion caused by uneven gravitation loading as a function of elevation angle. This is only a problem at the higher frequencies of $\mathrm{X}$ - and Ka-band. The surface is optimized for 45-deg elevation and falls off more or less symmetrically at the zenith and horizon. This is illustrated in Fig. 13, which shows the variation of X-band efficiency with elevation. For X-and Ka-band, there is almost no variation of efficiency with azimuth. However, for S-band (which has virtually no variation with elevation), there is both a calculated 


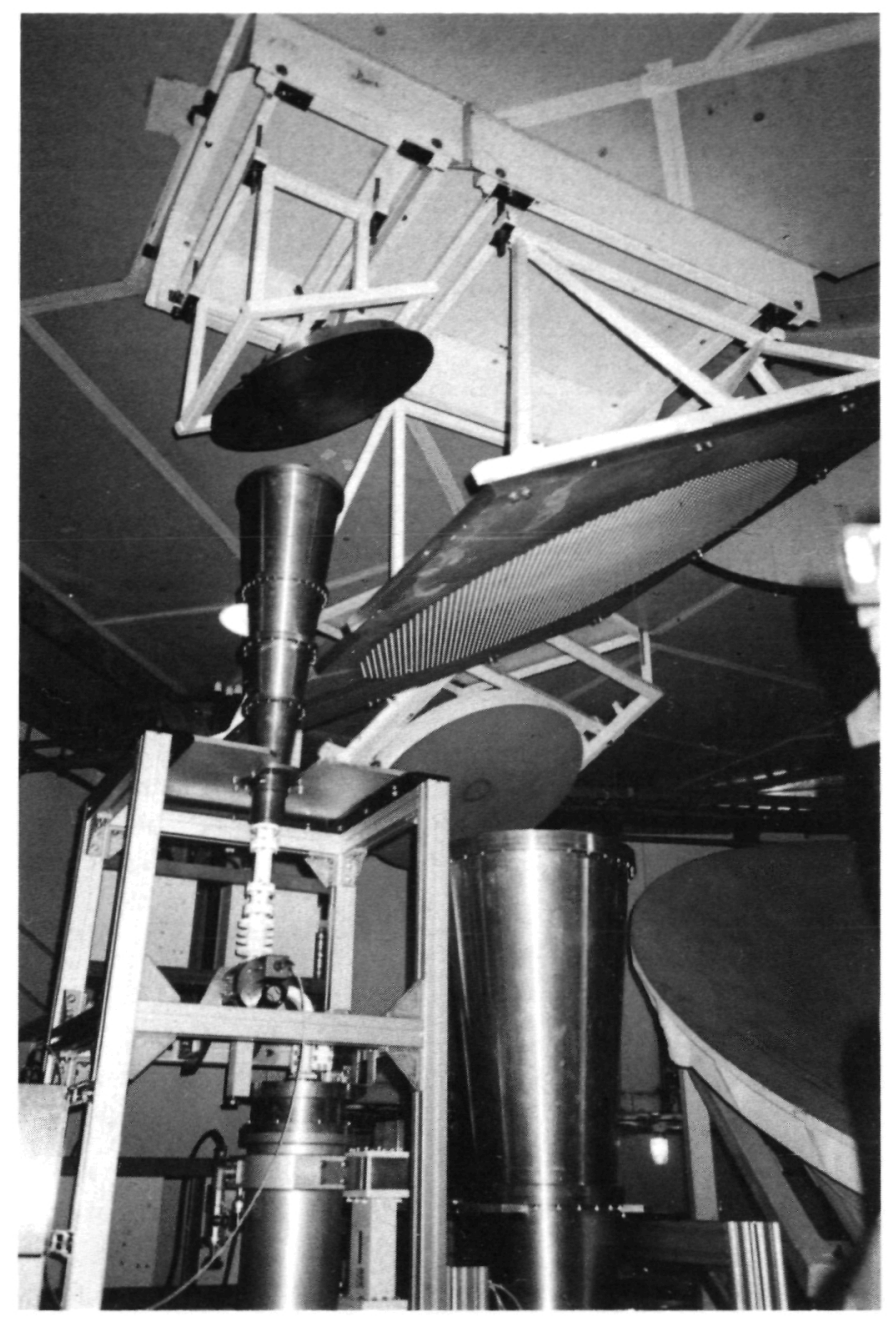

Fig. 12. Feed system installed in BWG antenna.

Table 2. Noise temperature predicts and measurements, $\mathrm{K}$.

\begin{tabular}{lcccc}
\hline \multicolumn{1}{c}{ System } & $\begin{array}{c}\text { S-band } \\
\text { predict }\end{array}$ & $\begin{array}{c}\text { S-band } \\
\text { measurement }\end{array}$ & $\begin{array}{c}\text { X-band } \\
\text { predict }\end{array}$ & $\begin{array}{c}\text { X-band } \\
\text { measurement }\end{array}$ \\
\hline LNA & 8.3 & 8.72 & 12.0 & 14.09 \\
$\begin{array}{l}\text { Feed system } \\
\text { (including LNA) }\end{array}$ & 17.69 & 17.5 & 23.07 & 24.0 \\
Antenna (total) & 37.26 & 38.0 & 32.9 & 33.0 \\
\hline
\end{tabular}

and a measured variation with azimuth, as shown in Fig. $14 .^{2}$ This is due to the rotation of the ellipse with respect to the upper BWG mirrors. At S-band, there is more asymmetry at the ellipse output than at X- or Ka-band, probably attributable to more of the ellipse being illuminated due to the lower-gain horn.

\footnotetext{
${ }^{2}$ The measured data are courtesy of Michael Klein, Jet Propulsion Laboratory, Pasadena, California, May 1995.
} 


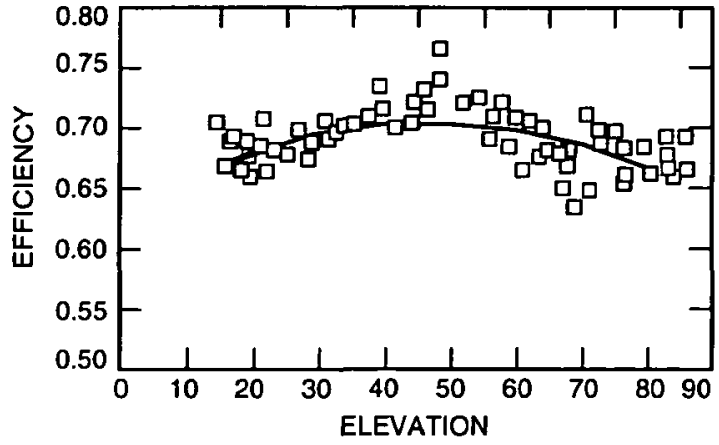

Fig. 13. Measured $X$-band efficiency variation with elevation.

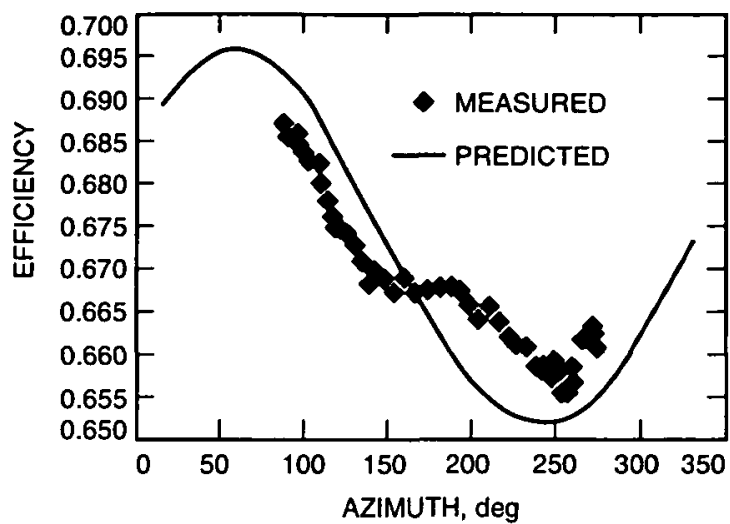

Fig. 14. S-band efficiency variation with azimuth.

\section{Conclusions}

A novel solution to the S-band design problems in a geometrically designed BWG system has been demonstrated. The proposed design was implemented as part of an S-/X-band feed system in the DSS-13 antenna located at Goldstone, California. The measured and predicted performances of the feed systems and the overall antenna agree very closely.

\section{Acknowledgments}

This task owes its successful completion to the teamwork and dedication of the people who were involved. Many people from different organizations have made valuable contributions by offering their expertise and hard work. In particular, the authors would like to acknowledge J. Chen and P. Stanton for dichroic plate analysis; T. Otoshi, M. Franco, and S. Stewart for feed and antenna measurements; J. Fernandez and J. Loreman for repair and testing of the S-/X-band LNA; D. Ball, R. Bryant, and D. Ohashi for mechanical design; and M. Gatti for funding support.

\section{References}

[1] M. Mizusawa and T. Kitsuregawa, "A Beam-Waveguide Feed Having a Symmetric Beam for Cassegrain Antennas," IEEE Trans. Antennas Propagat., vol. AP-21, pp. 844-846, November 1973.

[2] T. Veruttipong, J. R. Withington, V. Galindo-Israel, W. A. Imbriale, and D. Bathker, "Design Considerations for Beam-Waveguide in the NASA Deep Space Network," IEEE Trans. Antennas Propagat., vol. AP-36, pp. 1779-1787, December 1988.

[3] T. Veruttipong, W. Imbriale, and D. Bathker, "Design and Performance Analysis of the New NASA Beam-Waveguide Antenna," National Radio Science Meeting, Boulder, Colorado, p. 59, January 1990. 
[4] T. Veruttipong, W. Imbriale, and D. Bathker, "Design and Performance Analysis of the DSS-13 Beam-Waveguide Antenna," The Telecommunications and Data Acquisition Progress Report 42-101, January-March 1990, Jet Propulsion Laboratory, Pasadena, California, pp. 99-113, May 15, 1990.

[5] S. Rengaragan, V. Galindo-Israel, and W. Imbriale, "A Study of Amplitude and Phase Shaping Effects in Beam Waveguides," IEEE AP-S International Symposium, Dallas, Texas, pp. 1502-1505, May 1990.

[6] W. A. Imbriale and R. E. Hodges, "The Linear-Phase Triangular Facet Approximation in Physical Optics Analysis of Reflector Antennas," Applied Computational Electromagnetic Society, vol. 6, no. 2, pp. 74-85, Winter 1991.

[7] Y. Rahmat-Samii and V. Galindo-Israel, "Shaped Reflector Antenna Analysis Using the Jacobi-Bessel Series," IEEE Trans. Antennas Propagat., vol. AP-28, no. 4, pp. 425-435, July 1980.

[8] A. C. Ludwig, "Near-Field Far-Field Transformations Using Spherical-Wave Expansions," IEEE Trans. Antennas Propagat., vol. AP-19, no. 2, pp. 214-220, March 1971.

[9] D. A. Bathker, W. Veruttipong, T. Y. Otoshi, and P. W. Cramer, Jr., "BeamWaveguide Antenna Performance Predictions With Comparisons to Experimental Results," IEEE Transactions on Microwave Theory and Techniques, vol. 40, no. 6 , pp. 1274-1285, June 1992.

[10] T. Cwik and J. C. Chen, "DSS-13 Phase II Pedestal Room Microwave Layout," The Telecommunications and Data Acquisition Progress Report 42-106, April-June 1991, Jet Propulsion Laboratory; Pasadena, California, pp. 298-306, August 15, 1991.

[11] S. A. Brunstein, "A New Wideband Feedhorn With Equal E- and H-Plane Beamwidths and Suppressed Sidelobes," Deep Space Network, Space Programs Summary 37-58, vol. II, Jet Propulsion Laboratory, Pasadena, California, pp. 6164, July 1969.

[12] P. D. Potter, Improved Dichroic Reflector Design for the 64-Meter Antenna $S$ - and X-Band Feed Systems, JPL Technical Report 32-1526, vol. XIX, Jet Propulsion Laboratory, Pasadena, California, pp. 55-62, February 15, 1974.

[13] J. R. Pyle, "Cutoff Wavelength of Waveguides With Unusual Cross Sections," IEEE Transactions on Microwave, Correspondence, vol. MTT-12, no. 5, pp. 556557, September 1964. 